\title{
Die 'Lex Hadriana de rudibus agris' nach einer neuen Inschrift.
}

\author{
Von A. Schulten.
}

Zwar ist der in der Bearbeitung der letzten Inschrift von den afrikanischen 'saltus' ${ }^{1}$ ) ausgesprochene Wunsch, es möchte einmal das Original der Lex Manciana und der Lex Hadriana gefunden werden, noch nicht

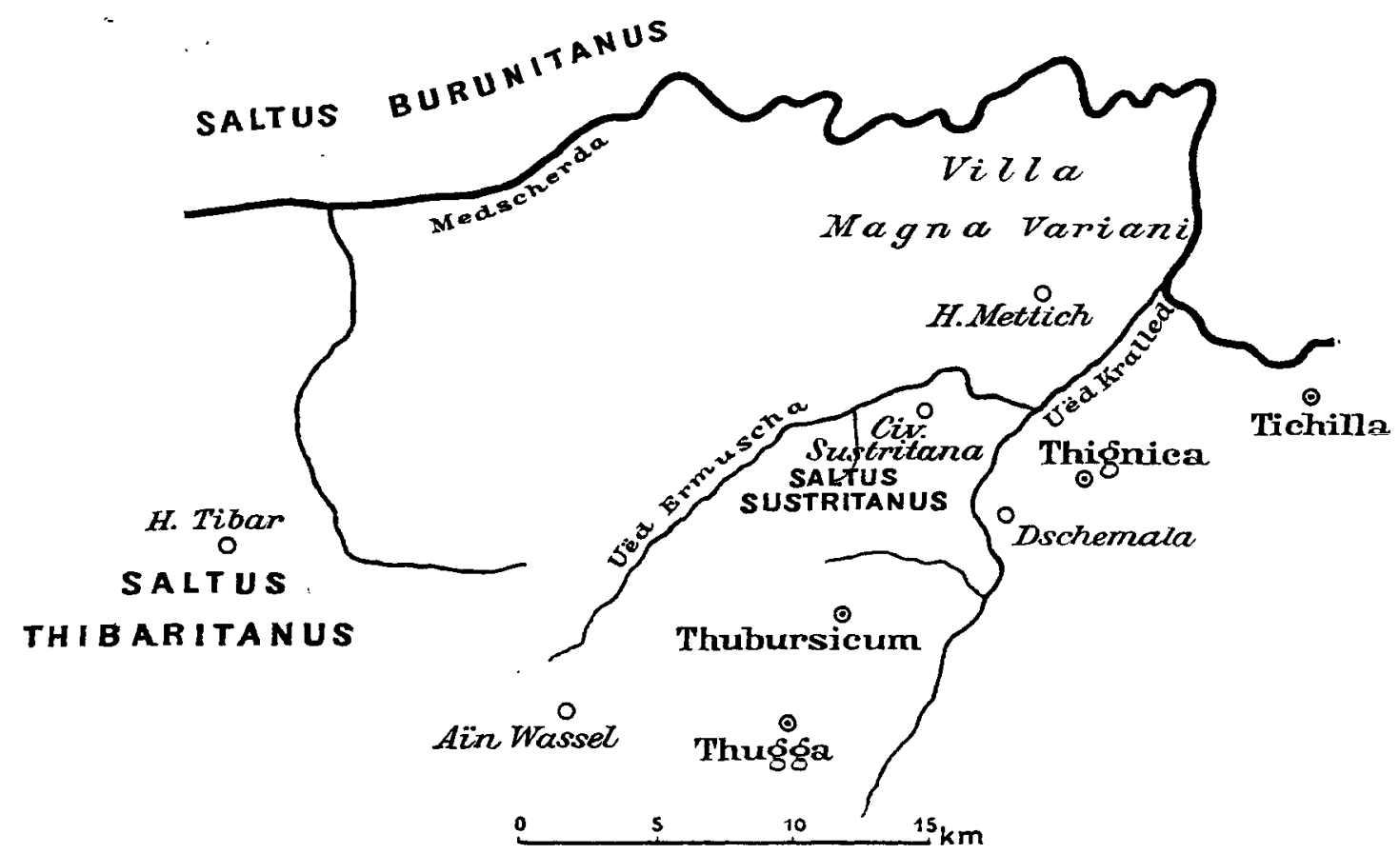

in Erfüllung gegangen, aber auch für die neue Inschrift können wir dankbar sein, denn sie ermöglicht eine vollständige Herstellung der von mir Hermes 1894, $204 \mathrm{f}$. herausgegebenen Inschrift mit dem Auszug aus der lex Hadriana de rulibus agris et iis qui per $\mathrm{X}$ annos continuos inculti sınt. Es ist bereits die sechste Urkunde ${ }^{2}$ ) von den Domänen des Bagra-

1) Die 'Lex Manciana', eine afrikan. Domänenordnung (Abh. d. Gött. Ges. d.Wiss. 1897).

2) Ich führe die früheren und ihre Ausgaben an, schon weil ich mich im folgenden auf sie beziehe. Es sind 1) das Dekret des Commodus über den Saltus Burunitanus von Sûk el Khmis (Mommsen, Hermes 1880), 2) die Lex Hadriana de rudibus agris von Aïn Wassel (Schulten, Hermes 1894, 204), 3) die Lex Manciana von H. Mettich (Schulten, a. Anm. 1 a. O.), 4) das Fragment von Aïn Zaga, .) das von Gasr Mezuar, die ich Hermes 1894, 204 behandelt habe. 
dastals, und der neue Fund steigert die Hoffnung auf eine vollständige lex saltus, die er selbst nicht erfüllt. Gefunden wurde der Stein von Herrn Carcopino, einem jüngeren Mitgliede der Ecole de Rome, die ja, seit in Italien das ' ' Italia farè da se' auch auf die Archäologie angewandt wird, ihren Schülern in Afrika ein neues, fruchtbares Arbeitsfeld angewiesen hat, bei einer epigraphischen Streife in der Umgebung von Aïn Tunga, Thignica, wo Herr Carcopino Ausgrabungen veranstaltete. Der Stein lag im Bett des Flusses Uëd Kralled, in der Nähe der Quelle A ïn el Dschemala, 6 km sw. von Aïn Tunga (s. die Karte S. $188^{1}$ ). Das wertvolle Denkmal hat im Bardomuseum neben den Steinen mit der Lex Hadriana und Manciana, mit denen es sich so eng berührt, Aufstellung gefunden. Der glïckliche Finder hat seinen Fund in den Mélanges der Ecole de Rome 1906, 365-481 mit einem ausfïhrlichen Konmentar herausgegeben, dem eine Photographie der Inschrift beigegeben ist. Er hatte die Freundlichkeit, mir noch vor der Veröffentlichung die Photographien und seine Abschrift zukommen zu lassen. Ich zitiere Herrn Carcopinos Arbeit im folgenden mit C. und der Seitenzahl der Mélunges ( ${ }_{n}$ C. p. ..").

Der Stein ist oben wie durch langjährige gleichmässige Einwirkung des Wassers abgerieben, unten dagegen abgebrochen (C. p. 386). Die sich hieraus ergebende Wahrscheinlichkeit, dass oben wenig,. unten viel fehlt, wird durch den günstigen Umstand bestätigt, dass wir mit Hülfe der Ara legis Hadrianae (I. von Aïn Wassel) imstande sind, Kolumne II und III herzustellen. Verteilt man die fehlenden Stücke der Lex Hadriana auf beide Kolumnen mit Berücksichtigung der Zeilenlänge, die gewöhnlich 28-30 Buchstaben beträgt, so ergibt sich, dass diese Kolumnen 18-21 Zeilen hatten, dass also in beiden, die noch 11 Zeilen aufweisen, unten 7-10 Zeilen fehlen, während oben Kol. III keine, Kol. II eine Zeile eingebüsst hat. Daraus ergibt sich derselbe Verlust für Kol. I und IV, von denen 11 und 9 Zeilen erhalten sind. Auch hier können oben nur wenige (1-2), müssen unten bei $I$ etwa 8 , bei IV etwa 10 Zeilen fehlen.

Ich gebe zunächst den Text der neuen Inschrift, wobei ich in der Lesung des Erhaltenen nur selten (II 7; IV 5;9), häufig dagegen in der Herstellung der Lücken von C. abweiche. In Kol. II und III ist das nur in der Ara legis Hadrianae Erhaltene kursic, das nur in der neuen Inschrift Erhaltene fett, das beiden Texten Gemeinsame in gewöhnlicher Schrift gedruckt. Fehlende Buchstaben sind durch [..] und eben so viele Punkte, zuviel gesetzte Buchstaben durch \langle\rangle , ausgelassene und $\mathrm{Ab}$ kürzungen durch ( ) bezeichnet. In wie ẃ eit die Ergänzung kleiner Lücken des neuen Textes durch den alten bestätigt wird, ist aus einer Vergleichung der beiden Texte zu ersehen und deshalb nicht besonders angegeben. Das Ende der Kolumnen der Ara legis Hadrianne ist durch !! bezeichnet.

1) Die Kartenskizze S. 188 beruht auf der von Herrn Carcopino p. $4: 57$ mitgeteilten. 
(Es fehlen ca. 2 Zeilen).

$\begin{array}{rl}1 & \text { [coloni] ... tuani. hogamus, procurato- } \\ 2 & \text { [res, per pro]videntiam vestram, quan } \\ 3 & \text { [vice Ca]esaris praestatis, velitis nobis } \\ 4 & \text { [et utilitat]i illius consulere, dare no }\langle\mathrm{s}\rangle- \\ 5 & \mathrm{~b} \text { [is eos agros] qui sunt in paludibus et } \\ 6 & \text { in silvestribus instituendos olivetis } \\ 7 & \text { et vineis lege Manciana condicione } \\ 8 & \text { [s]altus Neroniani vicini nobis. Cu[m] } \\ 9 & \text { [ed]eremus hanc petitionem nostr[am, } \\ 10 & \text { [fu]ndum suprascriptum N[eronia-] } \\ 11 & \text { [num i]ncrementum habit[atorum ....] }\end{array}$

(es fehlen etwa 8 Zeilen).

II

1 iubeas. Sermo procurato[rum im]-

2 p. [C]aes. Hadriani Ang. Quia Cae[sar. n. pro]

3 infatigabili cura sua, per qu[am adsi]-

4 due (pro) humanis utili[ta]tibus exc[ubat, om]-

5 nes partes agrorum, quae tam oleis au[t]

6 vineis quam frumentis aptae sunt [ex]-

7 [c]oli iubet, itcirco permissu $\langle\mathbf{m}\rangle$ prov[id-

8 [en]tiae eius potestas fit omnibus e[tia]-

9 m eas partes occupandi quae in c[ent]-

$10\lceil\mathrm{u}]$ ris elocatis saltus Blandiani $e[t \mathrm{U}]-$

11 [de]nsis (et) i[n illi]s partibus sunt [quae ex]-

12 saltu Lamiano et Domitiano iuncta(e)

13 Thusdritano sunt nec a conductoribus

14 ex[er]centur, i(i)sque qui occupaverint

15 possidendi ac fruidi eredique $s[u]$ o

16 relinquendi in ius datur quod $e(s) t$

17 lege Ha(dri)ana comprehensum de rudi-

18 bus agris et iis qui per $X$ an $(n)$ os con-

19 tinuos inculti sunt. Nec ex Blandic-

20 no et Udensi saltu maiores partes

21 fruc $\|[$ tunum]

III

1 [q]ua[m coloni] ob s[u]mm[am Caes. cle- $]$

2 [mentiam is qui lo]ca neglecta a co[ndu]-

3 [ctoribus] occupaverit, (sed) qua[e hi da]- 
Die 'Lex Hadriana de rudibus agris' nach einer neuen Inschrift. 191

4 [re sole]nt, tertias partes fructuu[m],

5 [dabit. De] eis quoq(ue) regionibus qua-

6 [e ex] Lamiano et Domitiano

7 [saltu] iunctae Tuzritano sunt

8 [tantundem] dabit. De oleis quas quisq[ue]

9 [e possessorib]us [po]suerit aut oleas[tr]-

10 [is quas inseruerit] c[aptor]um fruct[uum]

11 nu[lla pars] decem proximis annis exige-

12 t[ur]; set nec de pomis septem annis pro-

13 ximis; nec alia poma in divisionem umq-

14 uam cadent quam quae venibunt a posses-

15 soribus. Quas partes aridas fructum

16 quisq[ue] debebit dare, eas proximo quinq-

17 uennio ei dabit, in cuius conductione

18 uryr(os) occupaverit, post it tempus rationi.

IV

(es fehlt 1 Zeile ${ }^{1}$ )

1 [C]arinus et Dor[yph]orus Primige[nio

2 [s]uo salutem. Exemplum epistulae scrip-

3 tae nobis a Tutilio Pudente, egregio viro,

4 ut notum haberes, et it, quod subiectum est,

5 [c]eleberrimis locis propone. Verridius

6 Bassus et Ianuarius Martiali suo salut[em].

7 Si qui agri cessant et rudes sunt [aut sil]-

8 vestres aut palustres in eo salt[uum trac]-

9 [tu, v]olentes lege Mancia[na colere ne prohibeas] (es fehlen etwa 10 Zeilen).

I 3 C.: [nomine] Cuesaris.

I 4 C.: [maiestat]i, aber utilituti ist drastischer (s. S. 203).

I 11. Ich möchte ergänzen : incrementum habit[atorum cepisse et maiores fructus reddere vidimus]. Auf das Gesuch folgte die Antwort des a rationibus Tussidius Clemens. Sie endet mit iubeas in Kol. II 1.

II 3 Aïn Wassel: curator per.

II 4 (pro) ist aus der I. von Aïn Wassel einzusetzen.

II 5 Ä̈n Wassel: quam tam.

II 7 ist offenbar nicht mit C. per missum providentiae eius zu lesen, sondern permissu $\langle\mathrm{m}\rangle$ zu emendiren. Was soll hier, wo doch die procuratores die kaiserliche Bewilligung ausführen, 'noch ein anderer 'missus'? ?

II 10 Aïn Wassel : Blandiani Uden[sis . . . i]n illis. Es könnte hier Uden[sisque et i] $n$ geschrieben gewesen sein, ebensogut kann aber in dem schlecht redigierten Text hinter Blandiani das et ausgefallen sein, welches der neue Text gibt.

1) Etwa: Exemplum epistulae proc. e. v.: . . . 
II 13 Die Inschrift von Aïn Wassel gibt Thusdritanus, die nene Inschrift Tuzritanus. Es muss heissen Thustritanus (s. unten S. 198).

III 1. Da in Zeile $2 \mathrm{f}$. steht: [ . qui lo] ca neglecta a co[ndu]ctoriluts occupaverit und, wie wir aus der Inschrift von Aïn Wassel sehen, vorausging: nec... maiores partes fructum, so muss in Zeile 1 offenbar die Kategorie genannt gewesen sein, der die Okkupanten gleichgestellt werden: die Kolonen. Auf nec maiores partes fructutum wird also zunächst gefolgt sein: quam coloni. Zu quan passen die Buchstabenreste am Anfang der Zeile .. VA .. Man wird also [q]un]m [colomi. .] herstellen dürfen. Auf VA folgt eine Lücke von 7-8 Buchstaben, dann drei Buchstaben, von denen der erste sicher ein $\mathrm{O}$, der zweite wohl ein $\mathrm{B}$, der dritte ein $\mathrm{S}$ ist, dann fehlt wieder ein Buchstabe; es folgt MM und es fehlen am Ende der Zeile 7-8 Buchstaben. Zeile 1 stellt sich also so dar: . VA.... ... OBS . MM ........ Ich glaube, dass das so herzustellen ist:

(Nec ... maiores partes fructuum)

1 [q] $\mathbf{u a}[\mathrm{m}$ coloni] ob $\mathbf{s}[\mathrm{u}] \mathrm{mm}$ [am Caes. cle- $]$

2 [mentiam is, qui lo]ca neglecta a co[ndu]-

3 [ctoribus] occupaverit, (sed) ${ }^{1}$ ) qua[e hi da]-

4 [re sole]nt tertias partes fructuu[m]

5 [dabit.

Die Herstellung ob summam Cresaris clementicam passt sowohl zu den Buchstabenresten als zum Raum und setzt einen überaus passenden Be-

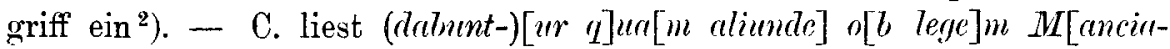
nam]. Das ist epigraphisch nicht möglich. da hinter OB und vor MM nicht LEGE gelesen werden kann.

III, 10 habe ich olens[tris quus inseruevit] geschrieben (Carcopino: oleastris inseruerit), denn der wilde Oelbaum dient nicht zum Propfen, sondern wird gepfropft (vgl. Lex Manciana $\$$ 9: qui insemerit olerstra ..).

IV 5 ist offenbar yropone (Carcopino: proponi) zu lesen. Der Stein, auf dem e und $\mathbf{i}$ oft nicht zu unterscheiden sind, erlaubt, der Satzbau fordert diese Lesung.

IV 7 Carcopinos [si qui sil]restres ist eine zu künstliche Konstruktion.

IV 7. Hinter rules sunt setzt C. eine starke Interpunktion; falsch, denn silrestres cut palustres gehört als nähere Erklärung von rudes zu diesem Begriff.

IV 9 ist rolentes (nicht volcntis) zu lesen, das Objekt zu einem nachfolgenden Verbum. Ich möchte [colere ne prolibeas] vorschlagen.

Die vier Kolumnen folgen sich in der obigen Ordnung anf dem Stein, wenn man wie selbstverständlich von links und rechts liest. Aber nur für II und III, die inhaltlich zusammenhängen, ist auch die innere Reihenfolge von vorne herein klar, für den Anschluss der anderen beiden an sie

1) Ein Wort ist ausgelassen auch II 4 (pro).

2) Auch in der auf Hadrian zurückgehenden lex metalli Vipascensis (\$ 2 der neuge. fundenen Tafel. s. u. S. 204) wird die liberalitas sacratissimi imperatoris Hallriani gerühmt. 
Die 'Lex Hadriana de rudibus agris' nach einer neuen Inschrift. 193

bestehen zunächst die drei Möglichkeiten II + III, IV, I; IV, I, II + III; I, II + III, IV. C. (p. 389) führt aus, dass die erste Kombination unmöglich sei, weil die in I enthaltene Petition nicht hinter dem in II + III enthaltenen Bescheid stehen könne. Er verweist auf die Analogie des Dekrets des Commodus; man kann ferner die Inschrift von Skaptoparene (Bruns, fontes ${ }^{6}$ p. 248) anführen, wo auch das Libell voransteht. Aber es gibt auch Fälle, wo in der Tat die Aktenstücke nicht in ihrer chronologischen Folge mitgeteilt werden, und dem Gesuch anderes vorausgeht: so in der Sache der Orcisteni (Bruns, fontes ${ }^{6}$ p. 157). Entscheidend ist, dass der mit iubeas (II, 1) endende Brief eine frühere Kolumne voraussetzt und die beiden folgenden Kombinationen fordert. Die zur Widerlegung der zweiten Kombination von $\mathrm{C}$. angeführten Grïnde sind nicht genügend, es gibt aber bessere. Der in Kol. IV erwähnte Brief des Oberprokurators Tutilius Pudens muss vorher, also, da II III von dem Sermo eingenommen werden, in Kol. I mitgeteilt gewesen sein, denn von dem anderen Brief (des Verridius Bassus) wird im Gegensatz zu ihm gesagt, dass er 'unten' mitgeteilt sei. Also ging Kol. I der Kol. IV voraus. Dass zwischen beiden II III stand, ergibt sich auch noch aus folgendem. Der Brief des Pudens (in I) muss einleitend unmittelbar vor dem Sermo, vor II III, gestanden haben, denn der Sermo, die den Kolonen gïnstige Urkunde, kann nur an Ende des Briefes stehen: als Anlage, als die Norm, nach der die Prokuratoren verfahren sollen. Also stand I nnmittelbar vor II III. Also ist die Reihenfolge I, II, III. IV die richtige.

Für den Brief des Tutilius ist in der unteren Hälfte der Kol. I vollkommen Raum, denn hier fehlen, wie wir oben sahen, etwa 8 Zeilen. Das dem sermo procc. vorausgehende inbeas in II, 1 würde dann das letzte Wort des Briefes sein. Man darf sich nicht wundern, dass der Brief iuberts sagt, während doch nach IV, 1 f. der Brief des Tutilius an die beiden Prokuratoren Carinus und Doryphorus gerichtet war. Die beiden stehen offenbar nicht an Rang gleich, sondern sind wie die beiden Prokuratoren der Inschrift vom saltus Burunitanus und der Lex Manciana, von denen der erste Ritterrang hat, der zweite Freigelassener ist ${ }^{1}$ ), der erste procurator tract us (Carthaginiensis), der zweite ein Unterprokurator und Freigelassener, wohl ein procurator regionis, sodass Tutilius sehr wohl nur den ersten anreden, aber der Brief auch den andern, als Gehülfen des ersten, betreffen konnte ${ }^{2}$ ).

1) Dekret des Commodus IV 10 : exemplum epistulae egregii viri: Tussanius Aristo et Chrysanthus ..; Lex Manciana I, 3: (lex) data a Licinio Maximo et F'eliciore Aug. lib(erto) procc. S. Hirschfeld. Vervaltungsbeamten ${ }^{2}$ S. 126.

2) C. (p. 405) hält beide für Freigelassene (soclass der erste nicht proc. tractus sein könnte) und procuratores regionis wegen der Namen. Aber vor Carinus kann in der fehlenden Zeile der zweite Name gestanden haben. Zwei procuratores regionis nebeneinancler sind ferner ein Unding, was auch C. sehr wohl sieht, inclem er den unmöglichen Ausweg findet. der betreffende Saltus habe zu zwei Regionen gehört (p. 406). Die Auffassung des Carinus und Doryphorus als procuratores regionis hat 
Ganz ähnlich wird in der burunitanischen Inschrift der Brief, der 'Tussanius Aristo (der proc. tractus) et Chrysanthus (der Unterprokurator) AnIronico suo salutem' beginnt, bezeichnet als exentplum epistulae e(gregii) $v($ iri $)$, als Brief des einen, des Tussanius. Da die uns bekannten procurcttores tractus ${ }^{1}$ ) die 'tria nomina' oder wie der Tussianus Aristo des burunitanischen Dekrets mit Fortlassung des Praenomen die beiden Hauptnamen führen, ist vor Carinus sein Nomen zu ergänzen. Zu dieser Auffassung der beiden Prokuratoren Carinus et Doryphorus als procurretor tractus und Gehülfe passt, dass sie nur noch eine Instanz unter sich haben, den Primigenius, der wie die anderen nur ein Cognomen führenden Beamten: der Andronicus des saltus Burunitanus, der Patroclus Augyg. lib. pror. der Ara legis Hadrianae, procurator saltus ist ${ }^{2}$ ).

Wenn aber Carinus procurator tractus ist, so kann der vir egregius Tutilius, der ihm Befehle gibt, nur der nächst höhere und zugleich höchste Finanzbeamte, der (procurator) 'a rationibus' sein, der ja stets vir egregius ist ${ }^{3}$ ). An ihn würde sich also die Petition der Kolonen gewendet haben. Dem widerstrebt deren Anrede "procurrtores" nicht, denn der a rationibus hat einen Unterdirigenten neben sich $^{4}$ ) und wie die beiden in dem Erlass an den Aufseher der Marcussäule vom J. $193^{5}$ ) zusammen verfügen, so können sich hier die Kolonen auch an beide zusammen gewendet haben. Eine analoge Erscheinung, dass bald der procurator tractus allein, bald zusammen mit seinem Gehülfen genannt wird, haben wir eben kennen gelernt. Für meine Auffassung des Tutilius spricht ferner, dass auch die Kolonen des saltus Burunitanus sich an die höchste Instanz wenden. Das ist in jenem Falle der

zur Folge, dass C. den Tutilius zum procurator tractus macht. Da er aber richtig erkennt, dass der 'sermo procuratorn'm' nur von dem proc. a rationibus und seinem adiutor herrühren kann, so bleibt ihm nun nichts übrig als Verridius Bassus und Ianuarius (IV, 5) für diese zu halten. Aus diesen Irrtümern ergibt sich für C. auch eine ganz verkehrte Auffassung des Dekrets des Commodus (p. 422). Fr hält den proc. tractus Tussanius Aristo und Chrysanthus für den a rationibus und seinen Ge-

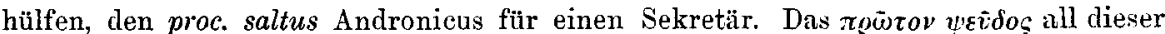
Verwirrung scheint grewesen zu sein, dass C. (p. 420) den Brief des Verridius Bassus et Ianuarius (IV $5 \mathrm{f}$.) mit dem vorher (IV, $1 \mathrm{f}$.) erwähnten des Tutilius identifiziert. obwohl der Brief des Verridius mit 'et id quod subiectum est' deutlich von jenem unterschieden wird und sich selbst durch die Verschiedenheit der Verfasser unterscheidet. Da er an der Stelle, wo er den Brief des Tutilius erwartet, einen solchen des Bassus und Ianuarius findet, hält C. den Tutilius für einen Unterbeamten des Ianuarius, der nur den Brief seines Chefs weitergegeben habe, sodass sein Brief auch als der des Ianuarius habe bezeichnet werden können, und fasst diesen als a rationibus, den Tutilius als proc. tractus auf.

1) S. meine Schrift Die röm. Grundherrschaften (1896) S. 62.

2) Das zeigt die Analogie des Provincialis Aug. lib. proc. vom Saltus Massipianus und des Theoprepes Aug. lib. proc. at praedia Galliana proc. saltus Domitiani. S. Hirschfeld, Verwaltungsbeamten 2 S. 133.

3) S. Hirschfeld, a. a. O. S. 29; 125

4) S. Hirschfeld, a. a. O. S. 32 f. - 5) Bruns, fontes ${ }^{6}$, p. 303. 
Die 'Lex Hadriana de rudibus agris' nach einer neuen Inschrift. 195

Kaiser, musste aber hier der Stellvertreter des Kaisers, der a rationibus sein, weil sich ja die Kolonen nicht an den Kaiser, sondern an die procuratores gewandt hatten, an den a rationibus und Gehülfen. Ferner passt ein Ausdruck wie 'per providentiam vestram quam [vice] Ca esaris praestatis' am besten auf den wirklichen Vertreter des Kaisers, den höchsten Finanzbeamten. Es entspricht der Centralisation der kaiserlichen Verwaltung, dass wie überall und speziell im Fall der burunitanischen Kolonen so auch hier die Entscheidung von der höchsten Instanz, nicht etwa rom proc. tractus, ausgeht.

Wir sahen, dass der sermo procurator um imp. Hadriani eine Anlage des von Tutilius gegebenen Bescheids bildet. Stammt er deshalb anch, wie C. (p. 421) meint, aus dem Bureau des a rationibus, sind die procuratores des Sermo identisch mit den von den Kolonen angeredeten "procuratores": dem a rationibus und seinem Gehülfen? Nein. Auf eine Petition zu antworten und wichtige Entscheidungen zu treffen, ist gewiss Sache der höchsten Instanz, dagegen war eine blosse Mitteilung des bestehenden Rechts, wie sie der Sermo enthält, gewiss Sache der procurctores tractus und so gut wie, die beiden Prokuratoren, von denen die lex Iata ad exemplum legis Mancianae herrührt, es sind, werden die Verfasser des Sermo, der ja ebenfalls eine lex aul exemplum legis (Hadrianae) ist (Aïn Wassel I, 7), der procurator tractus und sein Gehülfe sein. Dafür spricht auch die Analogie des Scrmo zu den litterae procuratorum, quae sunt in tabulario tuo tract us Karthaginiensis der burunitanischen Urkunde: die ebenfalls ausser dem Bescheid der höchsten Instanz als Rechtsquelle angeführten Briefe der procuratores tractus.

Wer aber sind Verridius Bassus et Ianuarius, deren Brief an Martialis von dem proc. tractus Carinus und seinem Gehülfen dem proc. saltus Primigenius zugleich mit dem Schreiben des Tutilius zur Publikation mitgeteilt wird? Eine weitere Anlage des Briefes des Tutilius kann der Brief des Ianuarius nicht sein, denn eine solche müsste an den Sermo anschliessen, könnte nicht nachher selbständig mitgeteilt sein. Vielmehr wird der Brief des Verridius offenbar selbständig von Carinus produziert, als Analogon zu dem von Tutilius angeführten sermo procurctorum. Die beiden Verfasser des Briefes "Verridius Bassus et Ianuarius" werden wie . Carinus et Doryphorus der erste procuratortractus, der zweite ein Unterprokurator, etwa proc. regionis, sein und der Adressat Martialis entsprechend dem Primigenius des Carinusbriefes proc. saltus. Der Parallelismus von "... Carinus et Doryphorus Primigenio suo salutem" und "Verridius Bassus et Ianuarius Martiali suto salutem" ist evident und hat sein genaues Gegenstück in dem "Tussanius Aristo et Clerysanthus Andronico suo salutem " der Insehrift von Suk-el-Khmis. War Verridius Bassus procurator tractus, so erklärt sich auch, dass Carinus seime Korrespondenz besitzt, die sich ja im Bureau des tractus befand. Ferner ist der Adressat des Verridius wegen seines einen Namens, Martialis, offenbar proc. saltus. 
Auch deshalb muss Verridius proc. tractus sein. In den Briefen des Carinus und des Verridius Bassus haben wir dann solche Briefe von procurctores tractus, wie sie im Dekret des Commodus zitiert werden (s. oben), vor uns.

Der Geschäftsgang, den die Petition der Kolonen unserer Inschrift macht, ist also folgender. 1) Die Kolonen richten ihr Gesuch an die Centralbehörde, die 'procuratores': Tutilius Pudens und seinen uns unbekannten Gehülfen - wie die burunitanischen Kolonen an den Kaiser -; 2) Tutilius übermittelt seine den Kolonen günstige Entscheidung dem proc. tractus Carinus und seinem Gehülfen Doryphorus, indem er die Anwendung des sermo proce. Hadriani auf den vorliegenden Fall und die Aufstellung dieses Sermo anordnet - wie im Fall der burunitanischen Kolonen das Reskript des Kaisers dem proc. tractus Tussanius Aristo und seinem Gehülfen Chrysanthus mitgeteilt wird -; 3) sendet der proc. tractus Carinus den Bescheid des Tutilius unter Beifügung einer analogen früheren Urkunde an den proc. saltus Primigenius zur Aufstellung (mopone) - wie Tussanius Aristo den Brief des Kaisers an den proc. saltus Andronicus - ; 4) stellt Primigenius die in dieser Angelegenheit produzierten Aktenstücke in chronologischer Ordnung zusammen: (1) die Petition (an Tutilius), 2) dessen Bescheid mit dem sermo procuratorum, 3) den Brief des proc. tractus Carinus mit dem Brief des proc. tractus Verridius Bassus und lässt sie so auf unserem Stein einhauen "). Ganz ebenso stellt Patroclus Auggg. lit. die Ara legis Hadrianae auf: . arrum legis diri Hadriani instituit et legem. infra scriptam intulit. Neben den von den Kolonen aufgestellten arae: dem Dekret des Commodus (feliciter consummata et dedicata scil. ara) und der Lex Manciana (hec lex scriptr a Lurio Victore Odilonis mag. etc.) haben wir also zwei von einem procurator saltus aufgestellte arae: die Ara legis divi Hadriani und unseren Stein ${ }^{2}$ ).

Nachdem der Zusammenhang der Inschrift aufgeklärt ist, lassen sich in die Lücken der Inschrift mehrere notwendig vorauszusetzende Begriffe einsetzen. Aus der Analogie der Ara legis Hndricunce und des Steins von H. Mettich dürfte sich ergeben, dass am Anfang des Steines, wo 2-3 Zeilen fehlen, eine Ueberschrift gestanden hat des Inhalts, dass der Prokurator Primigenius eine Ara mit den nachfolgenden Urkunden aufgestellt hat. Dann folgte wohl die Ueberschrift der ersten Urkunde, etwa: [ $p r$ titio colonorum saltus . .] tuani. Auf die Eingabe der Kolonen folgte am Schlusse von Kol. I der durch das Libell veranlasste Brief des Tutilius an den proc. tractus Carinus. Die Ueberschrift und Adresse muss etwa gelautet haben: exemplum epistulae Tutilii Purdentis viri egregii. Tutilius

1) Nach C. p. 390 hätten hier wie beim Dekret des Commodus die Kolonen die Inschrift hergesteilt. Dem widerspricht der clen proc. saltus zur Aufstellung anweisende Befebl (IV 5).

2) Die Benennung des Altars von Aïn Wassel als ara legis divi Hadriani: also als Altar eines Abstractum hat Analogien in ara adoptionis (Tacitus, ann. 1, 14), ara clementiae, amicitiae (ib. 4,74), ultionis (3. 18). Ich verdanke den Hinweis Wissowa. 
Die 'Lex Hadriana de rudibus agris' nuch einer neuen Inschrift. 197

Pudens v. e. . Carino suo salutem. Von dem Brief ist nur noch das letzte Wort inbeas erhalten, Dass iubeas nicht etwa noch zum Libell der Kolonen gehört, zeigt auch der Singular: die Kolonen wenden sich an eine Mehrheit (procuratores, praestatis, velitis). Da der proc. tractus den proc. saltus anweist, den Brief des Tutilius aufzustellen (IV 5), so könnte der letzte Satz des Tutilius gelautet haben: . . itaque sermonem procuratorum imp. Caes. Hadriani proponere iubeas. Ebensogut kann sich aber iubeas auch auf die Anweisung beziehen, dem proc. saltus Primigenius zu befehlen, er solle die Okkupation erlauben : . itaque procuratorem saltus .. ut volentes colere sinat iubeas. Da sich die Eingabe der Kolonen auf Oedland (prilustria et silvestrin), dagegen der von Tutilius ungeführte Sermo auf gutes vernachlässigtes Land bezieht, muss Tutilius in seinem Briefe gesagt haben, dass die Bitte der Kolonen durch die Lex Hadriana erfüllt werde, und dass dieses Gesetz die Okkupation nicht allein für Oed-, sondern auch für vernachlässigtes Pachtland erlaube, wie aus dem beifolgenden die betreffenden Bestimmungen mitteilenden Sermo der Prokuratoren zu ersehen sei.

Auf den Sermo, der den Anhang des Briefes des Tutilius bildete, folgte die Ueberschrift des in Kol. IV erhaltenen Schreibens des Carinus und Doryphorus, also: exemplum epistulae. . Carini v.e.

Der eigentliche Kern unserer Inschrift ist der sermo proce. Hadriani. Er soll das Okkupationsrecht der Kolonen regeln. Während der Sermo in der Inschrift von Aïn Wassel nur sermo procurrtorum heisst, erfahren wir jetzt, dass er von Prokuratoren des $\mathrm{Hadr}$ ian herstammt, also dem Hauptgesetz, der eigentlichen Lex Halriana, gleichzeitig ist. Mittlerweile hat der Anfang der Lex Manciana (lex) data .. ad exemplum legis Manciance meine Vermutung, dass in der Inschrift von Aïn Wassel I, $7 \mathrm{zu}$ lesen sei: . legem infra scriptam intulit (ad) exemplum legis Hadrianae in arn proposita(e), und die Darlegung, dass der Sermo ein Auszug ${ }^{1}$ i aus dem Hauptgesetz, eine "nach Massgabe" der lex H. gegebene Anwendung einiger Paragraphen auf einen speziellen Fall ${ }^{2}$ ) sei, bestätigt. Die neue Inschrift ist der dritte Fall, dass die kaiserliche Domänenverwaltung einen auf Grund der Hauptgesetze für den speziellen Fall zusammengestellten Erlass aufstellen lässt, der zweite Fall einer Aufstellung des sermo procc. Hudriani. Wir sehen, dass ausser den kaiserlichen Gesetzen die Verfügungen der Prokuratoren auf dem Gebiet der Domänenverwaltung eine grosse Rolle spielen. Auch die burunitanischen Kolonen berufen sich auf epistulae procuratorum.

Unsere Inschrift unterscheidet sich von der von Aïn Wassel nur

1) Ich habe selbstverständlich nicht, wie mir Vulić (Wiener Studien 1905, 138) unterschiebt, einen wörtlich en Auszug gemeint, denn selbstredend konnten die Namen der fünf Saltus sich nicht im Hauptgesetz finden.

2) Aehnlich sagen die Juristen actionem dare ad exemplum actionis illius ( $L$. 30 pr. D. 3,5$)$. 
durch ihre Ausführlichkeit. Während Patroclus nur den Sermo mitteilt, hat Primigenius auf unserem Stein auch die zur Aufstellung führenden Schriftstücke veröffentlicht. Wir kennen bereits ein anderes Beispiel für mehrfache Aufstellung derselben Urkunde im Bereich der Domänen. Der Stein von Aïn Zaga (s. o. S. 188) enthält das Reskript des Commodus des Steines von Sûk-el-Khmis. In keinem dieser Fälle braucht die längere Inschrift das Original der kürzeren zu sein, denn das Reskript des Commodus und der sermo procuratorum lagen im Original im Bureau der Domänenverwaltung und konnten direkt von verschiedenen Seiten kopiert werden. Die beiden Kopien stimmen im allgemeinen wörtlich überein, nur dass unsere Inschrift (Kol. III 5) eis, die von Aïn Wassel (Kol. III 4) his hat und jene Tuzritanus, diese Thusdritanus schreibt. Diese Variante ist interessant, da sie zugleich mit colonia Sustritana (C. p. 424) zeigt, dass das th der berberischen Wurzeln thu, tha etc., mit der soviele Ortsnamen beginnen, ähnlich wie englisches th gesprochen und deshalb bald mit $t$, bald mit s wiedergegeben wurde. Der Vergleich des Stadtnamens Sustritanus, wie zweimal bezeugt ist (C. p. 424), und der Schreibung saltus Thusdritamus auf dem Stein von Aïn Wassel ergibt als richtige Schreibung: Thustritanus. Ich verstehe nicht, wie C. die ganz verkehrte Schreibung Tuzritanus des neuen Steines adoptieren konnte.

Aus der neuen Inschrift ergibt sich, dass am Anfang von Kol. II und III des Steines von A ïn Wassel statt einer, wie man bisher annahm, 3 und 2 Zeilen fehlen, Der Kopf von Kol. II wird so hergestellt:

I, 14 tes agrorum, quae tam oleis

II, 1 [aut vineis quarn frumento aptae]

1 a [sunt, coli iubet, itcirco potestas]

$1 \mathrm{~b}$ [fit etiam eas partes occu-]

2 [pandi] quae in centu-

3 [riis elocat]is saltus Blandiani

Der Kopf von Kol. III so:

II 16 saltu maiores partes fruc-

III 1 [tuum quam coloni ob summam Caes. n. clemen-]

2a [tiam is, qui loca neglecta a conduc-]

2 toribus occupave[rit, sed quae hi so-]

3 lent tertias par[tes] etc.

Ich habe die Zeilen mit Berücksichtigung der Zeilenlänge der Inschrift, die in Kol. II und III meist 25-30 Buchstaben beträgt, verteilt. $\mathrm{Zu}$ der grösseren Lücke passt die Angabe des Finders der Inschrift. Dr. Carton, dass zu dem Stein noch ein (später verlorener) Aufsatz (mit Buchstabenresten) gehört habe ${ }^{1}$ ).

Durch das neue Exemplar des Sermo wird erst dessen Zusammenhang klar, der auch nachdem durch meine Herstellung ex[er]centur einiges licht

1) Revue Archéol. 19 (1892) p. 215. 
Die 'Lex Hadriana de rudibus agris' nach einer neuen Inschrift. 199

in den dunklen Sinn der Rede gebracht war, zumteil noch unklar blieb. Das rätselhafte quid (Aïn WasseI I 10) wird durch quice ersetzt und in der Lücke am Anfang von Kol. II erscheint ein von quia regiertes Verbum iubet.

Die durch die nene Inschrift ermöglichte Herstellung des Sermo führt zu manchen neuen Ergebnissen. Für den Sermo selbst lernen wir folgendes. Während es nach der ersten Abschrift so scheinen konnte, als ob die lex Hadriana, das dem Sermo zu Grunde liegende allgemeine Gesetz, sich selbst auf die fünf genannten Domänen bezöge ${ }^{1}$ ), sehen wir jetzt, dass der Sermo nur jenes allgemeine Gesetz auf diese anwendet, ganz wie die lex data ad exemplum 7. Mancianue die Lex Manciana auf die Domäne Villa Magna.

Die Kopie von Aïn Wassel stammt aus dem Anfang des 3. Jahrhunderts. Für die neue Kopie gewinnen wir durch den vollen Titel sermo procuratorum II a driani zunächst einen Terminus post quem. Daraus, dass der den Bittstellern in dem Bescheid mitgeteilte Sermo aus Hadrians Zeit stammt, folgt noch nicht, wie C. meint, dass auch die Petition aus dieser Zeit ist. Sie könnte eben so gut viel später sein, verwendet doch die Inschrift von Aïn Wassel denselben Sermo noch im Anfang des 3. Jahrhunderts. Aber es lässt sich auf andere Weise zeigen, dass unser Stein in der Tat aus hadrianischer Zeit stammt. Daraus, dass die Kolonen sich nicht auf die Lex Hadriana, sondern auf die Manciana berufen, folgt, dass Hadrians Gesetz noch nicht existierte oder jedenfalls ihnen noch unbekannt war. Da nun aber der doch offenbar bald erfolgte Bescheid schon auf der Lex Hadriana basiert, so kann die Petition nur kurz vor Erlass des neven Gesetzes verfasst sein. Dass die Lex Manciana noch unter Trajan im Gebrauch war, wussten wir bereits aus der Inschrift von H. Mettich.

Man wird sich die Entstehung des Sermo wohl so zu denken haben, dass der procurator tractus Carthaginiensis eine über die fünf Domänen an ihn ergangene Anfrage, wohl der Kolonen, mit Berufung auf die kürzlich erlassene Lex Hadriana in mündlicher Ansprache (sermo) beschied. Wie in der burunitanischen Inschrift die Kolonen ausser der Lex Hadriana auch litterac procuratorum zitieren, so hat dieser sermo procuratorum später als Rechtsquelle gedient und wird statt des Gesetzes selbst nicht allein von Tutilius Pudens unter Hadrian für eine Domäne der Gegend von Dschemala, sondern von Patroclus und unter Septimius Severus für eine Domäne der Gegend von Aïn Wassel als Norm aufgestellt. Dass der Sermo eine lex ist, so gut wie das Originalgesetz, war erstens von vorne herein klar, wurde zweitens durch die Bezeichnung des Sermo als lex Hadriana (Aïn Wassel: . . aram le gis divi Hadriani . . instituit) bestätigt und fand dann in der Analogie der lex data ad exemplum legis Mancianae eine neue Bestätigung.

1) Nach meiner Herstellung: quid Caesar n. . omnes partes agromum ... quae tam oleis [. quam frumento vacant $]$ quae in centuriis .. saltus Blandiani (et) Udensis . sunt... 
Für den Inhalt der Lex Hadriana lernen wir aus der Ergänzung des Sermo wenig Neues und ich kann auf meinen Kommentar der Inschrift von Aïn Wassel verweisen.

Es ist klar, dass die Verkündigung des kaiserlichen Willens im Sermo (Cuesar omnes partes ayrorum .. excoli iubet) aus der Lex Hadriana, die unten zitiert wird, stammt. Wir lernen also, dass unter den agri rudes, auf die sich die Lex Hadriana de rudibus agris et iis qui per $X$ annos continuos inculti sunt (II 17) bezog, sowohl Acker- wie Baumland begriffen war. Wir haben dieses allgemeine Gebot, dass alles fruchtbare Land zu bebauen sei, offenbar in der solchen prinzipiellen Aeusserungen dienenden Einleitung der Lex Hadriana zu suchen.

Dass die lex Hadriana de rudibus agris etc. auf den Domänen des Bagradas aufgestellt war, nicht etwa sonst irgendwo stand oder gar aus den Archiven hervorgeholt werden musste, lehrt das Dekret des Commodus: (III 14) : . . utpote cum in acre incisa et ab omnibus omnino undique versum vicinis visa.. Auch der Eingang der Inschrift von Aïn Wassel bezeichnet die Lex Hadriana wie etwas Bekanntes: .. (ad) exemplum legis Hadrianae in ara proposita(e). Das Gesetz war nach dem Dekret des Commodus auf Kupfer geschrieben und die Kupferplatten an einem Altar angebracht. wie ja auch diese Auszüge aus ihm, das Dekret des Commodus und die Lex Manciana. auf einer Ara stehen. Augenscheinlich betraf das Gesetz den ganzen Domänensprengel am mittleren Bagradas, denn es gilt 1) in dem n. des Bagradas gelegenen saltus Burunitanus (Dekret des Commodus), 2) auf den fünf in den Inschriften des Sermo procc. genannten saltus bei Thugga, 3) in der Gegend von Aïn Wassel und 4) auf dem saltus bei Thignica, dessen Kolonen auf der neuen Inschrift auftreten. Suk el Khmis ist von Aïn Wassel ca. 30, von Aïn Dschemala ca. 40, dies von Aïn Wassel ca. $20 \mathrm{~km}$ extfernt (s. die Karte S. 188). Man muss sich fragen, ob die Lex Hadriana nur für die afrikanischen Domänen oder gar nur für einen Teil derselben. oder ob sie für das ganze Reich erlassen war (so C. p. 478), ob sie eine private lex praerlics Catesaris. dirta oder ein staatliches Gesetz war. Die einleitenden Worte sind allgemein gefasst, nennen keine Gegend; allgemein ist auch der Titel. Beirles könnte auf ein Domänenstatut nicht gut zu passen scheinen. Das ist jedoch noch kein Beweis, und die Verschiedenheit sowohl der Kulturen wie der sozialen Verhältnisse im weiten Reich spricht gegen ein allgemeines Gesetz. Aber die afrikanischen Domänen insgesamt dürfte das Gesetz betroffen haben. Ihre Verhältnisse wird der Kaiser während seiner afrikanischen Reise im Jahre 128 persönlich kennen gelernt und wohl bald darauf die lex de rudibus ayris erlassen haben.

Auf das Verhältnis des sermo procuratorum zur Lex Hadriana fällt nicht allein durch das neue Exemplar des Sermo, sondern auch durch den Auszug aus der Lex Manciana nenes Licht. Das neue, in 
Die 'Lex Hadriana de rudibus agris' nach einer neuen Inschrift. 201

einer anderen Gegend gefundene Exemplar des Sermo bestätigt meine Ausführung (Hermes 1894 S. 211), dass man solche Auszüge aufgestellt habe, weil das Gesetz an einem weit entfernten Orte angebracht war. Die Mitteilung von Auszügen statt einer vollständigen Kopie erklärt sich offenbar aus dem grossen Umfang der Gesetze. Wir besitzen jetzt ferner das Analogon der , lex data al exemplum legis Mancianae', ebenfalls eines Auszugs aus einem grossen Domänengesetz.

Für die $\mathrm{L} e x \mathrm{M}$ ancian a gewinnen wir einige neue Daten. Wir sehen aus ihrer Anführung sowohl durch die Kolonen (I 7), als auch durch die Prokuratoren (IV 9). dass sie auch in dieser Gegend, s. vom Uëd Kralled, nicht nur in der Gegend, wo der Stein mit der Lex Manciana gefunden wurde, n. des Flnsses (vgl. die Karte S. 188), auf der Villa Magna Variani, Geltung hatte. Die beiden Fundorte liegen ca. $10 \mathrm{~km}$ auseinander. Man würde schon aus dieser Distanz, die nicht wohl auf einen einzigen saltus passt, folgern dürfen, dass sie in der Zeit Trajans und Hadrians für das ganze kaiserliche Domanialgebiet dieser Gegend galt, wie später das Gesetz Hadrians, also nicht das Statut einer einzelnen privaten Domäne war, wie 'Tontain behauptete. Nun bringt aber die neue Inschrift die volle Bestätigung meiner Ansicht, dass die Lex kaiserliches Domanialgesetz war, denn hier berufen sich kaiserliche Kolonen und Prokuratoren auf sie. Die Bitte der Kolonen: dare nobis eos agros qui sunt in paludibus et silvestribus instituendos olivetis et vineis lege Manciana ist begründet in den $\$ \$ 7$ und 8 des Gesetzes, von denen der erste die Anpflanzung von Weinstöcken an Stelle alter Reben, der zweite die von Oelbäumen auf Oedland betrifft.

Wir haben oben (S. 199) gesehen, dass die neue Inschrift unter Hadrian aufgestellt wurde, also kurz nach der unter Trajan geschriebenen Inschrift mit der Lex Manciana. Diese Gleichzeitigkeit wird durch äussere Anzeichen bestätigt. Die ursprüngliche Höhe des neuen Steins lässt sich auf $0,70 \mathrm{~m}$ berechnen: das ist die Höhe des Steines von H. Mettich. Die beiden Steine stimmen ferner in der Breite der Kolumnen - ca. 0,45 m - überein. Wichtiger ist die vollkommene Aehnlichkeit der Schrift, nur dass die Inschrift von $\mathrm{H}$. Mettich einige kursive Formen hat, die der neuen Inschrift fehlen.

Wichtiges können wir der neuen Inschrift entnehmen für das Ve rhältnis der Lex Hadriana zu der älteren Lex Manciana.

Daraus, dass Tutilius Pudens die auf die Lex Manciana basierte Bitte der Kolonen durch Hinweis auf die Lex Hadriana erledigt, folgt doch wohl, dass die Manciana durch die Hadriana antiquiert w u rde. Dazu passt auch der Inhalt der Lex Hadriana, so weit wir ihn kennen. Auch sie betrifft das unbestellte Land, unà die dem Anbauer auferlegten Leistungen sind dieselben wie in der Lex Manciana: die partes tertice, die operce bince. Weil sie jene hierin wiederholt, antiquiert die Lex Hadriana die Lex Manciana. Indem sie aber auch das von den Conductoren vernachlässigte Land betrifft, geht sie ïber das ältere Gesetz 
hinars. Ein sehr gewichtiger neuer Begriff ist das etiam in Kol. II 8. Die Kolonen sollen a $\mathrm{u} \mathrm{h}$ das an Conductoren verpachtete Land, falls die Conductoren dasselbe vernachlässigen, okkupieren dürfen. Die Tatsache als solche war bereits durch meine Herstellung ex[er]centur klargestellt, und - wir müssen es uns gestehen - da in der Lex Manciana nirgends von einem solchen Eingriff in das Recht der Grosspächter die Rede ist, hätte man bereits früher die von der Lex Hadriana eingeführte Neuerung erkennen mïssen. Jetzt aber wird sie uns eindringlich genug vor Augen geführt und Carcopino durfte den Unterschied zwischen Lex Hadriana und Lex Manciana nicht auch jetzt noch verkennen und meinen, die Lex Hadriana sei nur eine Anwendung der Manciana auf bestimmte saltus ${ }^{1}$ ), und in Kol. III 1 eine Erwähnung der Lex Manciana in die Lex Hadriana einschwärzen: maiores partes fructum [quam aliunde ob lege]m M[ancianam]. Etiam zeigt, worin sich die neue Lex Hadriana von der älteren Manciana unterschied. Die Manciana erlaubte nur die Okkupation von noch nicht bebantem Land (ager rutis), die Hadriana dehnte die Erlaubnis auf das verpachtete und liegen gelassene Land a us, sofern nur diese Vernachlässigung bereits zehn Jahre dauerte. Sie heisst "Lex H. de rudibus agris et i is qui per $X$ annos continuos inculti sunt". Der zweite Begriff enthält die Neuerung: die Lex Manciana kennt Okkupation nur auf ager rudis. Ihre $\$ \S 15$ und 16 , in denen auch von Heimfall nach einer Wartefrist die Rede ist, haben nicht das Geringste mit dem neuen Okkupationsrecht zu tun ${ }^{2}$ ). Sie handeln von Land, welches der $0 \mathrm{kkupant}$ unter Kultur genommen und dann wieder liegen gelassen hat - es soll nach zwei Jahren vom Grosspächter dem Okkupanten entzogen werden -; die Lex Hadriana bezieht sich auf Land, welches der Grosspächter liegen lässt - es soll nach zehn Jahren dem Okkupanten offen stehen. Es könnte so scheinen, als ob in dem Satz (IV $7 \mathrm{f}$.) : . s si qui agri cessant et rudes sunt .. volentes lege Mancia[na colere ne prohibeas] die Prokuratoren sich für die von ihnen erteilte Erlaubnis zum Anbau von vernachlässigtem ${ }^{3}$ ) Pachtland (und Oedland) auf die Lex Manciana berufen. Aber offenbar gehört hier lege Manciana zu colere, nicht zu prohibeas. Die Lex Manciana bezog sich eigentlich nur auf Oedland - das zeigt ja die Inschrift von H. Mettich -, aber die Prokuratoren wenden sie wegen der A nalogi e des ager cessans zum ager rudis auch auf jenen an. Sie sagen

1) P. 466 : le sermo doit être considéré comme une adaption à des nouveaux saltus de cette lex Manciana. P. 469 nennt er die lex H. eine „réédition abrégée mais plus claire et plus douce" der Manciana.

2) Das ist in den früheren Bearbeitungen der Lex Manciana verkannt worden (s. meine Lex M. S. 33; Seeck S. 359). Auch Carcopino schreibt (p. 469): . . le ius colendi est attribué par la lex Manciana à tout cultivateur qui est venu occuper sur le fundus une parcelle abandonnée depuis deux années.

3) Cessare $=$ non exerceri. S. Columella II, 2: . . hic ager sive exercetur sive cessat. 
Die 'Lex Hadriana de rudibus agris' nach einer neuen Inschrift. 203

nicht: „Wer vernachlässigtes Land bebauen will, soll das dürfen, weil die Lex M. es erlaubt", sondern: "... soll das dürfen, und seine Rechte und Pflichten sollen nach der Lex M. geregelt werden ". Die von Hadrian in einem neuen Gesetz allgemein gegebene Erlaubnis zur Okkupation vernachlässigten Pachtlandes war also im einzelnen Falle schon von den Prokuratoren nach Analogie der Lex Manciana - ad exemplum 7. M. erteilt worden. Eine solche Uebertragung eines Gesetzes auf verwandte Fälle ist magistratisches Recht; das lehren die prätorischen actiones datae ad exemplum actionis illius.

Die Neuerung Hadrians war einschneidend genug. Okkupation unbenutzten staatlichen Landes kannte bereits die Republik: das alte Possessionsrecht auf dem ager publicus, und die Kaiser haben sogar gelegentlich okkupiertes Land geschenkt - so Vespasian die italischen subsecivc (Feldm. I, 133) und Nero den kyrenäischen ager publicus (Tac. ann. 14, 18), - was Hadrian tat, ist etwas ganz anderes. Er griff durch sein Gesetz auf das Pachtland der Grosspächter über. Es ist sehr wohl möglich, dass er dazu berechtigt war. Denn es ist sowohl allgemeines Recht, dass der Pächter das Pachtland ordnungsmässig zu bestellen ( $L .25 \$ 23 \quad D .19,2)$ und vor allem nicht liegen zu lassen hat ( $L .24 \$ 2$ cit.) und in der Lex Hadriana kann speziell den Conductoren die intensive Bestellung des bestellbaren Landes vorgeschrieben gewesen sein. Man darf deshalb Hadrians Eingriff durchaus nicht mit dem der Gracchen vergleichen, die den wirtschaftlich unabhängigen Possessoren ihren Besitz nahmen und dazu ohne Entschädigung.

Hadrian wird die Okkupation eingeführt haben sowohl im eigenen Interesse als in dem der Kleinpächter. Ihm lag an der Bebauung des Landes, denn je besser das Land bebaut wurde, um so höhere Renten konnten die Conductoren zahlen. Aber seine Massregel hatte auch einen sozialen Erfolg und wohl auch einen sozialen Zweck. Durch die Okkupation des nichtbestellten Landes gewannen viele kleine Leute eine Existenz. Dieser soziale Zweck wird in dem Sermo der Prokuratoren sogar als die Hauptsache hingestellt: quia Caesar . pro infatigabili curc per quam adsidue pro humanis utilitatibus excubat. . omnes partes ayronum .. cotere iubet, aber freilich fängt die Sprache kaiserlicher Gesetze bereits früh an gleissnerisch zu werden. Die Kolonen ihrerseits heben auch den Nutzen, den der Kaiser aus der Okkupation ziehen werde, hervor: velitis nobis [et.utilitati] illius consulere (et) dare nobis [eos agros] .., wo wohl statt C.' maiestati, besser ein drastischeres utilitati steht. Man kann die soziale Seite des Gesetzes sehr wohl mit den Reformen der Gracchen vergleichen. Wenn aber Hadrian und die gracchischen Brïder Aehnliches wollten, so sind die Mittel höchstens dann ähnlich, wenn die Pächter nicht zu intensiver Kultur verpflichtet waren. Freilich auch Hadrian brach Privatrecht um höherer Interessen willen. Aber ex hat 
eine viel glücklichere Hand gehabt als die beiden Gracchen, die doch schliesslich die Bodenreform nur durch die Störung alles Besitzes erreichten. Hadrians Eingriff in das überdies noch fragliche Recht der Grosspächter, das gepachtete Land als Weide zu nutzen, schädigte diesen nicht nur nicht, sondern verschaffte ihm obendrein noch eine Mehreinnahme; denn das ihm zufallende Drittel von den Ernten, die der Okkupant erzielte, war jedenfalls mehr wert als die bisherige Verwendung des Landes als Weide - es versteht sich, dass die Conductoren das gepachtete Land irgendwie nutzten, und nee exercentur passt auch auf nur als Viehtrift benütztes Land, da exerceri den Anbau bezeichnet. Es dürfte schwerlich ein gelinderes Mittel, fruchtbares, aber von den Besitzern vernachlässigtes Land in wirtschaftlicher und sozialer Hinsicht nutzbar zu machen, geben, als diese Lex Hadriana. Sie könnte dem italienischen Parlament zur Anwendung auf die römische Kampagna empfohlen werden, wo auch die Indolenz weniger Besitzer Tausende von Morgen guten Landes der Benützung entzieht. Gewiss, es handelt sich hier um Eigentümer, bei Hadrian um Pächter, der Eingriff in privates Recht würde stärker sein; aber der moderne Staat erlaubt sich ganz andere Eingriffe, als es der wäre, die Eigentümer der Campagna di Roma zu zwingen, entweder selbst zu bebauen oder andere bebauen zu lassen.

Dass Hadrian mit seinem Landeskulturgesetz Erfolg gehabt hat, lehrt schon der Umstand, dass dasselbe noch nach 150 Jahren als Norm galt. Unter Septimius Severus stellt ein procurator saltus eine Kopie des sermo procc. Hadriani, die Inschrift von Aïn Wassel, auf. Die Statistik der durch den Kaisernamen sicher datierten Inschriften (s. CIL VIII p. 1038) zeigt, dass mit Trajan und Hadrian der Aufschwung der afrikanischen Provinzen beginnt ${ }^{1}$ ) - mit diesen Kaisern werden die Inschriften plötzlich zahlreicher - : an diesem Anfschwung mag die Lex Hadriana ihren grossen Anteil gehabt haben. Viele Wohltaten habe der Kaiser den Afrikanern erwiesen, berichtet der Verfasser der Vita Hadriani (13, 4). Die lex de rutlibus ayris war sicher eine der grössten unter ihnen.

Durch einen glücklichen Fund in den portugiesischen Bergwerken fällt von dort her auf das afrikanische Statut neues Licht. Es heisst in der nengefundenen Tafel der lex metalli Vipascensis ${ }^{2}$ ), nachdem vorher Hadrian als der Gesetzgeber bezeichnet ist:

qui ex numero puteorum quinque unum aul vencum perduxerit in ceteris sicut suprascriptum est opus sine intermissione facito $: n i$ it a fecerit, alii occupandi $i$ us esto. Qui post dies XXV praeparationi impensarum datrs opus quidem statim facere coeperit diebus a utem continuis decem postea in opere cessaverit alii occupandi ius esto. Puteum a fisco venditum continuis sexmensibus inter-

1) Vgl. auch Toutain, Cités rom. de la Tunisie p. 154.

2) S. die Ausgabe von Cagnat im Journal des Savants 1906, 442. 
Die 'Lex Hatriana de rudibus agris' nach einer neuen Inschrift. 205

missum alii occupandi ius esto, ita ut cum venae ex eo proferentur ex more pars dimidia fisco salva sit ${ }^{1}$ ).

Wenn der Pächter des Schachtes den Schacht liegen lässt, fällt derselbe dem Okkupator (occupator steht etwas weiter unten) anheim. Das ist dieselbe Maxime wie in dem Statut für die afrikanischen Domänen. Selbst die Details berïhren sich: zehn Jahre lang ist das gepachtete Land gegen den Okkupator geschïtzt, zehn Tage der gepachtete Schacht. Wir gewinnen durch diese Uebereinstimmung zwischen den beiden Statuten einen Verwaltungsgrundsatz des Kaisers. Er wollte, dass die Pächter seiner Besitzungen, der Bergwerke wie der Landgüter, gut wirtschafteten, und die Säumigen wurden durch Arbeitswillige ersetzt, unbekümmert um ihr Besitzrecht. Das ist gewiss ein wichtiger neuer Zng im Bilde des grossen Reorganisators der römischen Verwaltung ${ }^{2}$ ). Ausser der 7ex Hadriana de rudibus agris und der lex metalli Vipascensis sind uns noch andere Gesetze erhalten, welche Hadrians Fürsorge für die Volkswirtschaft des Reichs offenbaren: das für Athen erlassene Gesetz (IG III, 38), in dem Hadrian anordnet, dass die Grundbesitzer eine Quote ihrer Früchte der Stadt Athen verkaufen sollen, die auf die Finanzen der Städte Astypalaia, Ephesos, Stratonikeia bezüglichen Reskripte ${ }^{3}$ ). Sie begründen das Lob, welches die Vita Hulriani der Fürsorge des Kaisers für den Staatshaushalt der Städte des Reiches spendett). Wenn die Prokuratoren in ihrem Sermo Hadrians infatigabilis cura, per quam adsidue pro humanis utilitatibus excubat rühmen, so ist das diesmal keine Phrase.

Ich behandle nun die Einzelheiten der neuen Inschrift. Am Anfang der ersten Zeile von Kol. I ist offenbar der Rest des Namens der petitionierenden Kolonen erhalten: ... tuani. Die Kolonen können adjektivisch als coloni . . tu(ni $i^{5}$ ) oder, wie gewöhnlich, nach dem Saltus als coloni saltus ..tuani bezeichnet gewesen sein. Wir kennen den Namen des Saltus nicht; einer der fünf im Sermo genannten Saltus und der im Libell genannte salturs Neronianus ist es nicht, da diese sechs Namen nicht auf .. tucanus enden.

Die gemeinsame Petition der coloni sultus ...tuctui ist ein neues Beispiel für die korporative, der Gemeinde angenäherte Organisation der kaiserlichen coloni saltus ${ }^{6}$ ). Unrichtig ist C.'s (p. 401) Schluss: weil die

1) Eine weitere Analogie: auch die Pächter der putei leisten partes, hier die Hülfte. Sie heissen ferner coloni (s. $\$ 6$ der neuen Tafel: colonus cuinsque putei) wie die Domänenpächter.

2) S. über Hadrians Reformen Hirschfeld, Die kais. Vervaltungsbenmten ${ }^{2}$ S. $476 \mathrm{f}$.

3) In Dittenbergers Sylloge $2385-387$.

4) K. 11 reditus quoque provinciales sollerter explorans, ut si alicubi quippiam deesset, expleret; K. 20: omnes publicas rationes ita complexus est, ut domum privatam quivis paterfamilias non satius norit. - 5) CIL VIII, 8812: coloni Kasturrenses.

6) $\mathrm{Zu}$ den früheren von mir. Grundherrschaften S. 103. zusammengestellten Zeugnissen ist seitdem der magister und defensor der Kolonen. für die die lex data ad exemplum legis Mancianae aufgestellt wurde, hinzngekommen. 
Kolonen zu keinem der im Sermo genannten Saltus gehören. seien es überhaupt keine coloni eines Saltus, sondern coloni pagi . . tuani, also kleine Bauern einer benachbarten Gemeinde, die in die Domäne aufgenommen zu werden suchen - ein hübscher neuer Zug, wenn er nicht pure Phantasie wäre! Ist es denn nicht ganz einfach, dass der für fünf Saltus erlassene Sermo auf einen anderen Saltus angewendet wird? Ich fürchte sehr, dass der C. p. 395 mitgeteilte Grenzstein, der zwischen dem Kaiser und einem anderen Territorium terminiert, und Merlins Auflösung der Inschrift

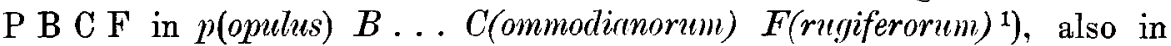
einen Gemeindenamen, Herrn $C$. einen üblen Streich gespielt hat.

Die Bitte der Kolonen, man möge ihnen Sümpfe und Waldland ,lege Manciana zur Anpflanzung mit Oliven und Wein überlassen, gründet sich auf die $\$ \S 7$ und 8 dieses Gesetzes. Dass die Kolonen nur diese beiden Kulturen vornehmen wollen, nicht auch Getreidebau, bezeichnet wohl den Vorrang, den $\mathrm{O}$ el - und We in b a u einnehmen. Auch die Lex Hadriana stellt sie vor das Getreide (II 5: tam oleis aut vineis quam frumento) und nicht minder der Sermo, indem er zuerst von Baumfrïchten (III $8 \mathrm{f}$.), dann von Getreide handelt (III 15 f.). Man sieht, zu Hadrians Zeit waren diese beiden die Hauptkulturen dieser Gegend Nordafrikas. Als die Lex Manciana erlassen wurde, scheint das noch nicht der Fall gewesen zu sein, denn hier werden Oel und Wein ziemlich am Ende behandelt und wird in der Zusammenfassung der Früchte in $\S 2$ zuerst Weizen, Gerste, Bohnen, dann Wein und Oel, zuletzt Honig genannt. Ich werde an anderem Orte zeigen, dass vieles auf ein hohes Alter der Lex Manciana hinweist. Dass sie in erster Linie den Ackerbau, in zweiter die Baumkultur behandelt, ist ein neues Argument dafür. Dass in Afrika der Ackerbau die ältere Kultur ist, ist bekannt genug. Um $400 \mathrm{v}$. Chr. bezog Karthago seinen Oel- und Weinbedarf aus Sizilien (Diodor 13,81) und noch Sallust sagt (Jugurthu 17) : ager frugum fertilis . . arbore infecundus.

In Afrika scheint sich also derselbe Wirtschaftsprozess vollzogen zu haben wie in Italien: der Uebergang von der extensiven Kultur, dem Getreidebau, zu den intensiveren Kulturen des Oel- und Weinbaus. In Italien ist dieser Wandel um die Mitte des 2. Jahrhunderts v. Chr., als Cato schrieb, vollzogen ${ }^{2}$ ), und zwar abgesehen von dem bei der Weidewirtschaft verharrenden Süden ziemlich allgemein. In Afrika hat sich Oel und Wein wohl zuerst im Norden, dem Gebiet des Bagradas, das fruchtbarer war und auf Absatz in der nahen Hauptstadt rechnen konnte, ausgebreitet, während der Süden, die Gegend der unendlichen Steppen, noch lange Korn-

1) Solche litterae singulares brauchen sich gar nicht auf das andere Territorium zu beziehen, bezeichnen vielmehr bei den Feldmessern (s. II p. 357) und auf dem Centurienstein in Toutains Cadastre de l'Afrique rom. (Mémoires présentées par divers savants à l'Académie d. Inscr. 1906) den Standort des Grenzsteins.

2) S. Gummerus, Der röm. Gutsbetrieb S. 18 f. 
Die 'Lex Hadrianu de rudibus agris' nach einer neuen Inschrift. 207

land blieb, ebenso wie Numidien, in dem noch zu Columellas Zeit (Col. 2, 2) nur Getreide gebaut wurde. Wir sehen aus der Schrift de bello Africano 36, dass im Jahre 46 v. Chr. diese Gegend: das Land um Thysdrus, der 'Sahel', später ebenfalls Olivenland ${ }^{1}$ ), noch Getreideland war. Aus dem Süden und aus Numidien wird zumeist das nach Rom gehende afrikanische Getreide, das um $70 \mathrm{n}$. Chr. zwei Drittel des ganzen Imports ausgemacht haben soll (Iosephus, bell. 2, 16, 4), und seit der Gründung Konstantinopels allein Rom versorgte, gekommen sein.

Auf die Bitte: velitis . . dare nobis . . folgen die beiden Begriffe: lege Manciana, condicione saltus Neroniani. Velitis dare lege Manciana lässt sich durch concedatis $l$. M. wiedergeben. Lege Manciana bezeichnet den Rechtsgrund der erbetenen Gewährung, ist ein Abl. causae ${ }^{2}$ ). Ebenso steht IV 9: volentes lege Mancia[na colere ne prohibeas). Gewöhnlich steht in einer solchen Verbindung $e x$ lege, so auch in der Inschrift von $\mathrm{H}$. Mettich: partes dare debebunt e l. M. (II 29) oder ex consuetudine l. MI. (I 23). Wo in dieser Inschrift ,lege ML.' steht, ist der Ablativ ,instrumental' (I 2 : permittitur lege MI.) wie auch sonst in der Sprache der Gesetze. Vergleichen kann man mit der Verbindung velitis dare nobis.. lege $M .=$ concedatis nobis $l$. $M$. Verbindungen wie lac lege oportet, nomina $h . l$. in albo proposita, $h . l$. accusare, mumerus decurionum per quem decreta h. l. facta sunt (l. Salpensana); comitia $h$. l. Thabere (l. Malacituna); 7. l. iurare (ib.), praedia h. l. vendere (ib.), bei denen auch der instrumentale Begriff fehlt, weshalb denn anch bei oportere sonst ex h. l. steht (Fraym. Florent. bei Bruns, fontes ${ }^{6}$ p. 120) und in der lex Malacitana auch comitia ex h. l. habere gesagt wird. Andererseits findet sich bei instrumentalen Verbindungen $e x h . l$, so in $e x h$. 7 . ius potestasque esto (neben $h .7$. ius potestasque esto in der 7 . Ursonensis) und ex h. l. civitatem consequi (neben $h .7$. civis factus in der lex Salpensana). Man sieht, der häufige Gebrauch der Formeln hat ihre Unterschiede verwischt, so dass hac lege und $e x h . l$. ziemlich durcheinander gebraucht werden.

Auch condicione saltus Neroniani ist kein korrekter Ausdruck: es müsste secundum condicionem s. $N$. heissen. Wie die Lex Manciana als Rechtsgrund, so wird das auf dem benachbarten Saltus herrschende und offenbar eben auf der Lex Manciana beruhende Okkupationsrecht als Präjudiz angeführt. Wie hier wird lex und condicio zusammengestellt von Cicero II Verr. $3 \S 40$ : . hoc est lege, edicto, condicione. Der folgende Satz bringt ein drittes Argument der Kolonen: das Gedeihen der benachbarten Domänen infolge des Okkupationsrechts. Der Satz möchte etwa so zu ergänzen sein: . . fundum s. $s$. $N[$ eronianum .. i]ncrementum habit[atorum cepisse et maiores reditus dare vidinus]. Den zweiten Begriff werden die Kolonen kaum anzuführen verfehlt haben, wie sie ja anch vorher I 4 auf das Interesse des Kaisers hinweisen.

1) S. Toutain, Cités rom. de la Tunisie p. 40.

2) S. Draeger, Histor. Syntax d. lat. Sprache I, 502 f. 
Die Begriffe in paludibus et in silvestribus bezeichnen das noch nicht unter Kultur genommene, wenn auch kulturfähige Land: das, was die Lex Hadriana ager redis nennt, wie denn auch IV 7 agri rudes durch sillestres aut palustres erläutert wird. Die Kolonen berufen sich für ihren Wunsch, es anzubauen, auf die Lex Manciana. Der betreffende Paragraph ist $\S 1$, in dem gesagt wird, dass die subsecira okkupiert werden dürfen: eos agros, qui s ub s e civ a sunt, excolere permittitur. Subseciva sind nicht nur die eigentlichen subseciva: die bei der Centuriation zwischen den äussersten limites und der Peripherie des centuriierten Gebiets entstehenden 'Schnitzel', sondern auch das im Inneren ganzer Centurien liegende unkultivierte und deshalb nicht adsignierte Land ${ }^{1}$ ). Vielleicht gehören hierzu sogar die überhaupt nicht centuriierten Landesteile, die sonst loca relirtı heissen ${ }^{2}$ ). denn sie werden ebenso wenig wie die subsecira adsigniert und stehen ihnen gleich: loca relicta .. sunt iuris subsecicorum sagt Frontinus. Es ist undenkbar, dass sie ron der Okkupation ansgenommen waren. Vielmehr wird der ganze arer rudis, alles noch nicht kultivierte Land, freigegeben worden sein.

Der Name des Saltus: Neronianus illustriert die bekannte Pliniusstelle (n. h. $18 \S 35$ ), derzufolge Nero sechs Grossgrundbesitzer getötet und ihre Güter, die die halbe Provinz Afrika einnahmen, konfisziert habe. Saltus Neroniamus bedeutet einen dem Nero gehörigen Saltus. Derselbe hatte offenbar seinen ursprünglichen Namen eingebüsst und den des kaiserlichen Erwerbers erhalten. Wie Hirschfeld bemerkt hat ${ }^{3}$ ), verraten uns vielleicht die Namen der im Sermo genannten Saltus: Lamianus, Domitiunus und Blandianus die Namen von dreien der von Nero getöteten Besitzer: eines Lamia, Domitius, Blandus. Für den letzten der drei hat das Carcopino (p. 436) nachgewiesen; der Saltus heisse wohl nach Rubellius Blandus, dem Vater des Rubellius Plautus, den Nero wegen seines Reichtums tötete ${ }^{4}$ ). Für den saltus Lamianus bezweifelt C. Konfiskation (p. 434). Aber daraus, dass die Güter des Aelius Lamia, des Freundes des Horaz, bereits unter Tiberius an den Kaiser fielen, folgt doch nicht, dass nicht die eines gleichnamigen Verwandten von Nero konfisziert wurden. C. (p. 435) bezieht den $s$. Domitianus wohl mit Recht auf Neros Tante Domitia, die der Kaiser, ebenfalls wegen ihrer Reichtümer, tötete. Die Villa Marnna Variani, für die der Auszug aus der Lex Manciana anfgestellt wurde, lehrt uns vielleicht einen vierten der sechs Grundbesitzer kennen, einen Varianus.

Daraus, dass die Lex Manciana auf dem saltus Neronianus galt, folgert C. (p. 437), dass die fundi Villuc Marmate Variani, für die jene lex arl exemplum legis Mancirmae gegeben wird, zum saltus Neronirnus gehörten. Welch verkehrter Schluss! Die Lex Manciana wird noch sehr viele andere

1) Feldmesser II, 393. - 2) Feldm. I, 22, 2.

3) Beiträge z. alten Gesch. 1902. 297.

4) Tacitus. ann. 14, 57. 
Die 'Lex Hadriana de ruditns agris' nach einer neuen Inschrift. 209

Saltus betroffen haben. C. übersieht, dass jener Saltus, Villa Marma Varianï heisst, also ein selbständiger Saltus ist, so gut wie der Neronianus.

Was wir bisher von der Lage des Saltus am mittleren Bagradas wissen, ist folgendes (s. Karte S. 188). Bei H. Mettich lag die Villa Magna Variani. Der Saltus dehnte sich vielleicht im N. und O. bis zum Medscherda, im Süden bis zum U. Kralled aus, was eine passende Begrenzung ergeben würde. Die civitas Sustritan im Winkel zwischen U. Kralled und U. Ermucha bezeichnet die Lage des nach ihr benannten sultus Thustritanus. An ihn grenzten, offenbar auf der offenen Seite, im SW. - der SO. ist Stadtgebiet von Thugga und Thubursicum - die saltus Domitianus und Lamianus, da ja Stücke von ihnen mit dem S. Thustritanus verbunden waren. In der Nähe sind saltus Udtensis und saltus Blandianus zu suchen, die mit den bisher genannten dreien eine Gruppe bilden. Ausser durch die Lage der ciritrs Sistritrna werden die fünf Saltus einigermassen dadurch fixiert, dass sie sowohl in der Nähe. von Aïn Wassel als auch in der Nähe des neuen Steines, also zwischen ihnen, gelegen haben müssen. Da der für sie erlassene Sermo an jenen Orten offenbar als nachbarliche Praxis angewandt wurde, wird Aïn Wassel im 0., Dschemala im W. an die Saltus gegrenzt haben. Durch die von C. p. 432 angeführte Inschrift kennen wir NO. von Aïn Wassel einen saltus Thibaritamus, benannt nach einer Gemeinde wie der Saltus Thustritanus und Udensis. 'Die drei Inschriften von Sûk el Khmis, Aïn Zaga, Gasr Mezuar lehren, dass sich n. des Medscherda der saltus Burunitrmus ausdehnte. Westlich von ihm, bei Schemtu, lag der saltus Philomusianus (CIL VIII, 14603). Je mehr Festpunkte wir gewinnen, desto mehr tritt die gewaltige Ausdehnung des kaiserlichen Domänengebiets am mittleren Bagradas hervor. Und auch die Zeugnisse von anderen afrikanischen Saltus mehren sich beständig $\left.{ }^{1}\right)$.

Die Bezeichnung centuriis elocatis (II 10) scheint zu bestätigen. was Seeck a. a. O. S. 335 ans der Verpachtung des ager vectiyalis nach Centurien gefolgert hatte: dass die Centurie oft als Pachteinheit gedient hat ${ }^{2}$ ). Da von den renturice elocatce gesagt wird . . nee exercentur; und sie später loca neglecta genannt werden, sind sie Kulturland. Daraus ergibt sich für den aus centuriis elocatis zu entnehmenden Gegensatz von nicht verpachteten Centurien die Wahrscheinlichkeit. dass diese das Oed-

1) Ich führe die nenesten an: term. n. VIII T. Anni Ampliati-term. n. VIII Iuliorum Flacci et Celsi aus der Nïhe von Tuburbo (W. von Tunis): Bull. du Comité 1904 p. XIX; Steine mit P.M.S.M. oder M.I.R.S.O.V. auf der einen und P. Aeli Macrini auf der anderen Seite aus dem Süden. der Gegend von Sbeitla: Bull. du Com. 1901, 112; in his praediis Maniliorum Arellionum auf einem Stein bei Medenine.

2) Auch bei Cato $(1 ; 3 ; 10)$, Varro $(1,18,4)$, Columella $(2,12,7)$ erscheint die Centurie als Wirtschaftseinheit. Cato operiert mit Feldern von 240, 120. 100 Iugera. Centurien von 240 Ing. sind durch die Feldmesser (T. 159. 14) bezengt: 120 Iug. ist also $1 / 2$ Centurie von 240: 100 ist 1/2 Centurie von 200 Iugera.

K I i o, Beitrigge zur alten Geschichte VII 2. 
land enthielten. Wir kennen solche Centurien aus der Adsignation, wo die ganzen nicht adsignierten ( $=$ hier , nicht verpachteten') Centurien centuriae vacuae, die nicht adsignierten Parzellen innerhalb einer ganzen Centuria subseciva hiessen (s. o. S. 208). Mit eben diesem Begriff wird denn auch in der Tat in $\$ 1$ der Lex Manciana das noch unbebaute Land bezeichnet: .. eos agros qui subseciva sunt excolere permittitur. Also sind die nicht verpachteten Centurien Oedland. Da sich in der Lex Manciana der Begriff subsecivo auf unbebantes Land bezieht, müssen umgekehrt die r!gri qui in paludibus et silcestribus sunt unserer Inschrift subseciva sein.

Für die centurice elocatue folgt auch aus ihrem Gegensatz zu den subseciva und den gleichbedeutenden palestria und silcestria, dass sie Kulturland waren: ,qua falx et arater ierit', wie es bei der Adsignation heisst. Wie nur Kulturland adsigniert wurde, wird also in f den Domänen nurKalturland verpachtet ${ }^{1}$ ). Den beiden Arten Landes entspricht denn auch der Doppeltitel der Lex Hadriana: de mulibus aypris et iis qui per $X$ annos continuos inculti sunt. Die rules ayri sind das noch nicht unter Kultur genommene aber kulturfähige Land, die silcestriı et palustria der subseciva, die agri qui per $X$ amos contimus inculti sunt das Kulturland der centuriae elorctue. Während die Lex Manciana nur die subseciva (\$ 1), die ayri qui in prahulibus et silcestribus sunt der Petition, freigibt, dehnt Hadrian das Okkupationsrecht auch auf das Kulturland, auf die verpachteten Centurien, aus. Was aber für Lündereien die postes (Kol. III 5 regiones) quee [ex] saltu Lamiano et Domitimn innatue Tuaritano sunt waren, war bisher noch unklar; die von mir a. a. O. S. 219 unten angeführte Erklärung befriedigt nicht. Innctur dürfte doch wohl für vicinae stehen, so dass nur die topographische Lage der betroffenen Teile der beiden Saltus angegeben wäre. Wären diese Parzellen wirklich dem saltus Thustritanus inkorporiert gewesen, was ja iunctre auch bedeuten kann, so versteht man nicht recht, warum sie noch als putes ex saltu Lamiano et Ullensi statt als Teile des Thustritanus bezeichnet werden. Die beiden Domänen würden also teils aus ager rutis, teils aus gutem und deshalb verpachtetem Land bestanden, und dieses letztere an den Saltus Thustritanus angegrenzt haben. Die Konstruktion: . partilus, quace [exx] saltu Lamiano .. iunctae Tuzritano sunt (statt . purtibus saltus Lamiani, qucce iunctce .. sunt) ist freilich etwas hart.

C. (p. 450) begeht den starken Fehler, ner. . exr[er]centur nur auf den zweiten Begriff (et in iis partibus etc.) zu beziehen, wo es doch evident ebenso gut zu dem ersten gehört, da, wie das Okkupationsrecht beide Kategorien betrifft, auch die Voraussetzung desselben, das non exerceri, auf beiden vorhanden sein musste. Er kommt so zu einem Gegensatz zwischen verpachteten Centurien und nicht bestelltem Land. was gar kein Gegen-

1) Elyenso war es auf dem ager publicus (Appian. b. cir. 1. 7). 
satz sein kann, da auch die zweite Kategorie deutlich Pachtland ist ${ }^{1}$ ). Der dem 'non exerceri' entsprechende Begriff [lo] ca ne gle ct a a co[nductoribus], III 2, belehrt jeden, der noch zweifelt, dass die agri qui non a conductoribus exercentur (nee .. a c.e. II 13) das von de $\mathrm{C}$ onductoren gepachtete und liegen gelassene Land sind, nicht etwa, wie C. meint, Land, welches die Conductoren nicht bestellten, weil sie es nicht gepachtet hatten. Negligere kann nur von dem gegen die dem Pächter obliegende Pflicht zur Kultur liegen gelassenen Land gesagt werden, nicht von Land, welches dieselben gar nicht zu bestellen brauchten.

Nicht minder verkehrt ist die Interpretation des Begriffs rationi am Schluss des Sermo (Aïn Wassel Kol. III, 18). Ratio $\varkappa \alpha \tau^{\prime} \dot{\varepsilon} \xi \circ \chi \eta^{\prime} \nu$ ist überall die ratio Caesaris, also auch hier. Dazu passt denn auch völlig der Zusammenhang. Die vom Okkupanten abgelieferten Quoten vom Getreide sollen während der ersten fünf Jahre dem Conductor, in dessen Pachtzeit die Okkupation fiel, gegeben werden, später dem Kuiser (post it tempus rationi). C. (p. 459 f.) meint, das sei unmöglich, da doch der Conductor Pächter aller Einkünfte sei, diese also nicht an den Kaiser gelangen könnten. Das ist er im allgemeinen gewiss, aber hier wird eben eine Ausnahme festgesetzt. Wer kann denn auch sonst die dem Conductor entzogenen Gefälle erhalten? C. meint, die Nachfolger des Conductor; , la

1) Auch Hirschfeld (Verwaltungsbeamten ${ }^{2}$ S. 130) scheint nec-exercentur in C.s Sinne zu verstehen, denn er stellt diesen Begriff in Gegensatz zu dem von den Conductores gepachteten Hofland, wie er ja auch bestreitet (S. 131), dass die Conductores den ganzen Saltus, also auch das Kolonenland, gepachtet hätten, und die : Kolonen nicht wie ich für Afterpächter der Conductores hält. Wenn aber die Conductores nicht bloss Pächter der von den Kolonen zu leistenden Vectigalien waren, sondern auch selbst wirtschafteten - wie die ihnen von den Kolonen geschuldeten opera ergeben -, so müssen sie doch wohl den ganzen Saltus gepachtet haben. Nur dann versteht man ferner, dass ihnen die Kolonen Frohnden schuldig sind, nicht aber, wenn sie nur ein Stück des Saltus, das 'Hofland' gepachtet hätten, also Parzellenpächter wie die Kolonen selbst gewesen wären. Die Frohnden lassen sich doch nur aus einem Hoheitsrecht, dem Besitz des ganzen Saltus, nicht aus der Pacht der Vectigalien ableiten. Auch werden die Conductores im Dekret des Commodus (III 29) conductores agrorum fiscalium also Pächter des Saltus selbst genannt. Hinzu kommt schliesslich die Analogie des ager vectigalis der Städte. Fr wird en bloc an Grosspächter verpachtet, und diese verpachten ihn in Parzellen weiter an Kleinpächter (Feldm. I, 116; Dig. 19, 2,53). Dass auf den Saltus die Conductores nicht erst selbst Parzellen abteilten, sondern offenbar die bestehenden Kolonen übernahmen, macht keinen Unterschied. Hirschfeld führt an, dass sich doch die Kolonen selbst als coloni domini nostri, rustici tui vernulae [et alumni] saltuum tuorum, also als Päichter des Kaisers bezeichneten. Das sind sie aber als Pächter des dem Kaiser gehörigen Landes auch dann, wenn zwischen sie und den Kaiser der Grosspächter tritt. Man könnte ferner einwenden, dass djch das Angebot des Iunius Martilianus 'in his praediis vectigalia locantur' (Mélanges 1898, 470) die Conductores als Pächter der Kolonenquoten bezeichne, aber vielmehr sind unter vectigalia alle Erträge der Domäne: nicht allein die Zinsen der Kolonen, sondern auch die des von den Conductores selbst bewirtschafteten Landes zu verstehen. 
ratio englobe toute la série de conductores qui se succéderont .. dans la ferme". Das würde zweifellos nach vorhergehendem ei . . in cuins conductione agrum occupavit, heissen: eis qui succedent, kann jedenfalls nicht mit 'rationi' wiedergegeben werden. Wie aber erklärt sich diese Bestimmung? Ein quinquennium ist die normale Pachtzeit und bleibt das auch dann, wenn die Pacht, wie offenbar die der Conductoren, faktisch weiterläuft. Nach Ablauf einer Pachtperiode gehören also die vom Okkupanten abzugebenden Quoten in der Tat zunächst dem Kaiser. Wird die Pacht verlängert, gehen sie eo ipso an den Conductor über. Mit den vorher behandelten Quoten von den Baumfrüchten ist das genau ebenso. Hier ist denn auch, was wohl zu beachten ist, nicht gesagt: conductori partes dare debebit, sondern allgemein: partes dare debebit.

$\mathrm{Zu}$ der dem Primigenius von Carinus zugehenden Anweisung zum Anschlag der mitgeteilten Akten: propone vergleiche man die analogen Befehle z. B. in Zuschriften der Praefecti Aegypti an die Strategen:

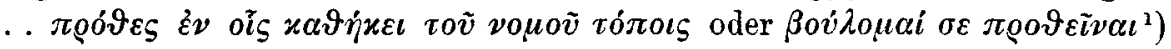
und in CIL III 13750 Z. 44 : propositum esse cura.

Ii qui occupaverint wird in der oben angeführten Stelle der lex metalli Vipascensis mit oc c u p a tor wiedergegeben. Der neue Rechtsbegriff ist willkommen. In der Inschrift von Aïn Wassel Kol. III 13 steht dafür possessor, was weniger scharf ist.

Aus der Bezeichnung des Tutilius Pudens (IV 3) als egregius vir lemen wir, dass dieser Titel nicht erst, wie er bisher schien ${ }^{2}$ ), unter Marcus und Verus, sondern bereits unter Hadrian vorhanden war. Da Hadrian den Rittern die Verwaltung des Reichs, soweit dieselbe kaiserlich war, übertrug ${ }^{3}$ ), der Schöpfer des ritterlichen Beamtenstandes ist, wir d man die Einfühung des Titels der Ritter 'viregregius' ihm zus chreiben dürfen.

In dem Brief des Carinus und Doryphorus (IV 2 f.) vermisst man das Verbum, von dem "t notum haberes abhängt, etwa misimus tibi, denn von propone kann es nicht abhängen, da der Zweck des Anschlags nicht ist, dass Primigenius das übersandte Schreiben kennen lernt, sondern, dass dasselbe zur allgemeinen Kenntnis gelangt und ferner propone ein habeas verlangt. Hier dürfte etwas ausgelassen und der Brief so herzustellen sein : (misimus tibi) exemplum epistulae .., ut notum habcres; (itaque exemplum. epistul(a) et id quod subiectum est . . propone.

1) S. Lafoscade, De epistulis imperatorum, magistratuum etc. 1902.

2) Hirschfeld, Verwaltungsbeamten ${ }^{2}$ S. 451.

3) S. Hirschfeld a. a. O. S. 478. 